\title{
Certificats de MOOC : débats passés et présents sur un objet en évolution rapide
}

Matthieu Cisel, laboratoire EDA, Université Paris-Descartes, 45 rue des Saint-Pères, 75006 Paris, France

Au début des années 2010, les MOOC acquièrent une notoriété, et leurs certificats, gratuits dans un premier temps, déferlent par centaines de milliers sur la Toile. D'aucuns crient à la mort des diplômes tels que nous les connaissons, et comparent le succès des différentes formations sur la base du nombre de certificats délivrés. Les débats sur ces questions prennent en ampleur, oblitérant souvent le fait que ces certificats recouvrent des réalités variées. Là où certains valident effectivement des acquis, beaucoup sont l'équivalent numérique de feuilles de présence. Après avoir montré et interprété cet état de fait sur la base de propos récoltés au cours d'une enquête menée auprès de concepteurs de MOOC, nous revenons sur les débats engendrés par les évolutions récentes de ces certificats. La gratuité a presque disparu, le secteur privé a commencé à se faire sa place dans l'univers $\mathrm{MOOC}$, ce qui ne manque pas de questionner le devenir des certificats nouvelle génération dans le milieu académique.

Les certificats des MOOC, gratuits dans un premier temps, ont été l'élément différenciant qui a conféré en peu de temps à ces dispositifs une notoriété mondiale. Rappelons en effet que des vidéos de cours universitaires étaient accessibles dans des Open Coursewares dès le début des années 2000 (D'Oliveira et al., 2010) et qu'elles ne constituaient plus en 2012 - l'année des MOOC - une grande nouveauté. Et pourtant, les conditions d'obtention de ces attestations de suivi, souvent nommées certificats, comme les controverses qui les entourent, restent encore relativement méconnues, les quelques études éparses sur la question ne fournissant pas à ces débats un ancrage empirique suffisant (Margaryan, Bianco, \& Littlejohn, 2015; Toven-Lindsey, Rhoads, et Lozano, 2015, Cisel, 2018b; Jordan, 2015). Nous nous proposons dès lors, dans cette contribution, de revenir sur deux des débats qui les entourent, en étayant nos propos par des extraits d'entretiens menés auprès de concepteurs de MOOC (Cisel, 2018a), pour conclure sur les controverses en émergence.

Le premier débat est celui des indicateurs de performance. II tire son importance du fait que les statistiques associées à ces cours sont pour beaucoup dans la notoriété et l'attention médiatique qu'ils ont reçues. Les premiers cours de Stanford sur l'Intelligence Artificielle ont conduit à la délivrance de plusieurs dizaines de milliers de certificats gratuits. Les détracteurs des MOOC n'ont pas manqué ensuite de souligner leurs faibles taux de certification pour défendre l'idée selon laquelle "ils ne marchaient pas ». Enfin, combien de fois a-t-on vu des enseignants mobiliser les taux de certification pour comparer le " succès " relatif des différents cours, et montrer que leur cours avait été un succès car ils affichaient des taux élevés?

Les échanges sur la question ont souvent cela de frustrant qu'ils négligent les différences parfois considérables entre les logiques de dispositifs. Comme nous allons le voir, là où certains concepteurs 
conçoivent le certificat de sorte à ce qu'il puisse sous certaines conditions donner lieu à des crédits académiques, d'autres le pensent comme un simple outil d'autoévaluation, ou l'équivalent d'une feuille de présence, n'ayant pas vocation à valider d'acquis. On peut dès lors se questionner sur la pertinence qu'il y a à mettre sur le même plan des indicateurs comme les taux de certifications, ou le nombre de certificats délivrés, pour des cours aux logiques aussi antagonistes.

Le second débat qui nous intéresse ici a agité un temps une partie conséquente de l'enseignement supérieur: il s'agit de l'impact supposé des certificats et attestations de MOOC sur le devenir des diplômes universitaires. Les chiffres annoncés régulièrement faisaient craindre à certains que les MOOC ne deviennent de nouvelles usines à diplômer, susceptibles de menacer le statu quo dans l'enseignement supérieur et de voir les établissements les plus fortunés s'imposer encore davantage sur la scène internationale via ces cours. Le recul dont nous disposons maintenant montre que ces craintes n'étaient pas fondées, d'une part car le modèle des MOOC des premiers jours n'était pas viable sur long terme pour des raison économiques, d'autre part car les certificats associés n'étaient en rien équivalents à des diplômes.

\section{Des certificats aux logiques variées}

Attachons-nous d'abord à montrer que les certificats peuvent avoir des niveaux de difficultés variés car ils répondent à des besoins tout aussi divers. II est des cas où le MOOC a été conçu en premier lieu pour être intégré dans un cursus académique (Cisel, 2018a), et les évaluations peuvent être pensées pour valider des acquis, avec un niveau exigeant pour un public novice. Dans ce type de situation, les exercices peuvent être identiques à ceux qui sont mobilisés dans un cursus académique, comme le souligne cet enseignant de mathématiques: "Certains exercices ont été donnés aussi en TD en présentiel. Ce cours a été inscrit [...] au niveau Master et ilsont fait des TD sur ces sujets. Certains des exercices sont rigoureusement les mêmes.»

Néanmoins, ce cas semble représenter l'exception qui confirme la règle. Le certificat a souvent vocation en premier lieu à inciter les participants à réaliser des évaluations qui n'ont pour seule fonction de mémoriser le contenu, au grand dam des apprenants qui aimeraient l'utiliser dans une logique de monstration de leurs " performances ". En d'autres termes, le certificat, à travers les exercices sur lesquels il se fonde, est pensé dans une logique " d'effet test " (Roediger et Karpicke, 2006) qu'il induit, c'est-à-dire qu'il vise à faciliter la mémorisation du contenu plus qu'à valider des acquis. Pour cet enseignant, la fonction principale du certificat réside dans la possibilité de pousser les participants à réaliser des quiz, simples à ses yeux, dont l'objectif est avant tout de s'auto-évaluer pour favoriser l'assimilation des connaissances :

On s'est posé la question du contrôle. Je pense qu'il est important de vérifier son niveau. L'intérêt du quizz noté, c'est de faire l'effort de se poser la question de savoir ce qu'on a ingurgité. On pose donc cinq questions que moi je trouve simples, avec trois réponses possibles. C'est plus pour faire l'effort de se poser la question, de se dire qu'est-ce que j'ai appris.

En d'autres termes, le certificat est souvent vu comme une carotte qui amène le participant à faire les exercices demandés, exercices qui ne permettent pas d'estimer le niveau d'acquisition de l'apprenant, quelle que soit la manière dont on le définit. Cette fonction de certificat comme simple " carotte " revient de manière récurrente dans les propos des enseignants. Certains acceptent de mettre en place cette certification simplement pour inciter les participants à terminer le cours : 
II semble que l'attestation soit une motivation pour certains apprenants ; ça correspond plutôt à une carotte pour l'apprenant. II faut aussi avouer que les QCM représentent la partie qui a été le moins travaillée. Pédagogiquement, il fallait mettre quelque chose et c'était important que l'apprenant ait l'impression à la fin du parcours d'avoir quelque chose en plus.

Selon cette logique, le certificat n'est pas pensé pour mettre en difficulté l'apprenant. Cet enseignant estime par exemple qu'il n'est proposé que pour reconnaître un investissement temporel ; il correspond alors à une forme de feuille de présence, qui atteste que les vidéos du cours ont effectivement été consultées.

Je comprends bien que ça demande du travail et que ça doit être valorisé d'une manière ou d'une autre. Je ne suis pas contre mais ce n'est clairement pas un diplôme. [...] Mais je trouve ça normal qu'on puisse donner un bout de papier quand quelqu'un a fait un effort substantiel pour suivre une formation au sens large. [...] C'est plus une feuille de présence parce que les quizz sont très simples et on exige $50 \%$ de bonnes réponses. II faut cliquer sur l'ensemble des vidéos.

La plupart des enseignants enquêtés voient le certificat comme une feuille de présence, une carotte, ou un l'outil d'auto-évaluation. Dès lors, pour ces enseignants, il reflète un engagement personnel plus que des acquis. Ils y voient avant tout le reflet d'une appétence pour l'apprentissage, d'une capacité de travail, comme l'illustrent les propos de cet enseignant de programmation :

Je trouve très bien que quelqu'un fasse spontanément tout seul la démarche d'apprendre et d'aller jusqu'à la certification. Je trouve que c'est le signe de quelqu'un qui aime apprendre et qui est volontaire. [...] la motivation est au moins aussi importante que le reste quand tu embauches quelqu'un.

Au passage, ce résultat est congruent avec ceux de l'enquête publiée par Kolowich (2013) ou par Evans \& Myrick (2015), qui avaient montré que la plupart des concepteurs de MOOC américains étaient sceptiques quant à la valeur du certificat qu'ils délivraient, et que dès lors aucun crédit universitaire ne devrait y être associé. Pour expliquer cet état de fait, il faut également garder à l'esprit le fait que les exercices associés au certificat sont quasi-systématiquement réalisés " à livre ouvert ", c'est-à-dire avec la possibilité d'accéder aux ressources pédagogiques du cours.

\section{Interpréter la diversité des situations : une nécessité pour se positionner dans le débat}

Comment interpréter cette diversité de fonctions attribuées au certificat? En premier lieu, par le plus faible niveau de contraintes pédagogiques auquel sont soumis les concepteurs. Rappelons que ces dispositifs échappent pour le moment à un certain nombre de règles. À l'exception des rares cas où le $M O O C$ est pensé dès le début pour être intégré dans un cursus académique, la principale contrainte en termes de conception pédagogiques est celle de la disponibilité des fonctionnalités portées par la plateforme hébergeant le dispositif. N'étant pas comptables devant l'institution du niveau de difficulté de l'évaluation, faute de délivrance de crédits universitaires, les enseignants disposent d'une grande latitude en matière de validation des acquis, liberté dont ils profitent différemment selon les motivations qui ont présidé au projet.

Quand la massification constitue un objectif prioritaire - pour satisfaire des financeurs ou pour diffuser un message notamment - la difficulté de l'évaluation est vraisemblablement tirée vers le bas et les taux de certification, sans doute vers le haut (Jordan, 2015). A contrario, quand le MOOC est plus ou moins 
articulé avec un cursus en place, que l'objectif soit l'hybridation ou la valorisation de ressources, l'incitation à adapter le niveau pour le grand public est moindre, ce qui ne manque probablement pas de tirer les taux de certification vers le bas. Dans ces conditions, on comprend aisément les problèmes que soulève la comparaison de la "qualité " de différents dispositifs sur la base de leurs seuls taux de certification : on compare des cours qui ne sont pas comparables par leurs fonctions. Par ailleurs, au regard de la manière dont est généralement conçu le certificat, on peut douter du fait que les diplômes des établissements d'enseignement supérieur aient été menacés de quelque manière que ce soit par l'émergence de ces cours. Comment parler de compétition entre certificats de MOOC et diplômes quand l'évaluation est le plus souvent adaptée pour le grand public, et réalisée " à livre ouvert ", l'attestation faisant office de récompense symbolique, de feuille de présence?

Certains veulent se fonder sur ce type de considérations pour clore le débat et affirment qu'il n'y a de ce fait aucun intérêt particulier à se pencher sur des indicateurs comme les taux de certification, ou le nombre de certificats délivrés. C'est noyer le bébé avec l'eau du bain. II est à nos yeux évident que ces indicateurs de performance ont de la valeur, et qu'ils constituent un objet scientifique légitime. II faut simplement prendre des pincettes dès lors que l'on s'autorise à réaliser des comparaisons ou à expliquer des phénomènes, il faut garder à l'esprit les différences que nous avons soulignées ici. En revanche, s'interdire toute réflexion en prétextant la complexité de la situation ne nous semble pas être une attitude scientifique valide.

D'aucuns diront que ces propos sont un peu datés, tant les certificats gratuits semblent avoir disparu du paysage - la France, avec le site France Université Numérique, constitue l'une des rares exceptions en la matière. Néanmoins, les évolutions de plates-formes américaines comme Coursera ou edX, avec l'essor des certificats payants et la réorientation vers la formation continue, ne rendent pas caduque la question des modalités d'évaluation et des conditions d'obtention du certificat. Elles ne font que la transformer, en floutant encore davantage la frontière qui existe en la matière avec les formations à distance payantes traditionnelles, et elles ouvrent naturellement sur de nouveaux débats.

\section{Les débats sur le renouveau des certificats de MOOC}

Les réflexions sur la certification dans les MOOC ont été centrées jusqu'à présent sur la fin de la certification gratuite. À quelques exceptions près, les plates-formes, l'une après l'autre, mettant un terme à la délivrance d'attestations et ne laissant bien souvent plus que les ressources vidéo du cours en libre accès. Dans quelle mesure la disparition de la gratuité a-t-elle affecté les exigences des évaluations associées ? On voit mal comment les logiques que nous avons identifiées plus tôt pourraient persister. En effet, quel apprenant rationnel mettrait la main à la poche pour obtenir l'équivalent numérique d'une feuille de présence?

Pour autant, il n'est pas sûr que les certificats de ces MOOC nouvelle génération, qui valent a minima quelques dizaines d'euros, soient équivalents à ceux de formations à distance académiques portant sur la même thématique, ces derniers se chiffrant en général en centaines d'euros. En d'autres termes, peuton comparer ces différentes formes de validation d'acquis ? Les services sont-ils similaires ? Quid de la correction individualisée des productions des apprenants ? II y a là un premier débat. Nous pensons à titre personnel que la chute des prix s'est accompagnée d'une dégradation sensible de la nature du service, en termes de quantité de contenus délivrés et d'individualisation de l'évaluation, mais les maigres données dont nous disposons sur les modalités de notation des apprenants ne nous permettent pas de trancher dans le débat à ce stade.

Le deuxième débat qui pourrait émerger à l'avenir est lié à la question des structures délivrant les certificats. Depuis quelques années, les entreprises ont investi l'univers des MOOC. La plate-forme Udacity a très tôt contractualisé, jusqu'à leur donner l'exclusivité, avec des entreprises comme Google, 
Facebook, Salesforce pour proposer des formations à leurs outils, entre autres choses. Ce type de dynamique a également percolé dans des grandes plates-formes américaines, et l'on peut voir aux côtés des cours de la Sorbonne ou du MIT des formations à Office dispensées par Microsoft. Là encore se pose la question de la comparaison par rapport à l'existant. Ce n'est pas la première fois que des entreprises dispensent en ligne ce type de certificats, mais tout porte à croire que l'évolution des prix - et sans doute également de la nature du service - pourrait avoir permis un passage à plus grande échelle.

Néanmoins, le débat qui nous semble le plus intéressant ici est avant tout celui de la reconnaissance de ce type de certificats dans le monde académique. Jusqu'à présent, la tendance a plutôt été à la compartimentation - ces certificats d'entreprise ont une certaine valeur dans le monde professionnel, surtout dans le monde anglo-saxon, mais ne semblent pas avoir pénétré outre mesure dans la citadelle du milieu académique. Mais peut-être les choses sont-elles amenées à évoluer maintenant qu'on les retrouve dans le même catalogue que les certificats d'université. On ne peut trancher définitivement sous prétexte que le secteur privé n'a pas sa place dans l'enseignement supérieur. Au vu du gain d'employabilité que permet la maîtrise des outils de ces entreprises, à commencer par la suite Office, la question a toute son importance. Dans la mesure où il est plus en plus fréquent pour des étudiants de faire reconnaître le suivi de $\mathrm{MOOC}$ dans leur cursus, nous sommes déjà confrontés à cette situation ; la réflexion sur le sujet a-t-elle suivi ?

Rappelons pour conclure que Linkedln a racheté Lynda.com pour près d'un milliard de dollars, soit le plus important rachat de l'histoire des technologies éducatifs. Et que la plate-forme, qui s'appelle désormais Linkedln Learning, va potentiellement délivrer des certificats à une échelle inégalée, et envahir la Toile, pour sans doute pénétrer un jour dans le monde académique. Nous estimons qu'il est temps désormais que le débat relatif au positionnement du milieu universitaire sur ce type de phénomène gagne en ampleur, d'autant plus si l'on veut éviter certaines dérives que cette pénétration de certificats d'entreprises dans le milieu académique ne manqueront pas d'entraîner.

\section{Bibliographie}

CISEL, M. (2018a). Les MOOC français à l'épreuve de la viabilité économique. Revue française des sciences de l'information et de la communication, (12). https://doi.org/10.4000/rfsic.3355

CISEL, M. (2018b). Une analyse automatisée des modalités d'évaluation dans les MOOC. International Journal of ELearning \& Distance Education, 33(1). Retrieved from http://ijede.ca/index.php/jde/article/view/1052

D'OLIVEIRA, C., CARSON, S., JAMES, K., \& LAZARUS, J. (2010). MIT OpenCourseWare: Unlocking Knowledge, Empowering Minds. Science, 329(5991), 525-526. https://doi.org/10.1126/science.11826962

EVANS, S., \& MYRICK, J. G. (2015). How MOOC instructors view the pedagogy and purposes of massive open online courses. Distance Education, O(0), 1-17. https://doi.org/10.1080/01587919.2015.1081736

ROEDIGER, H., \& KARPICKE, J.. (2006). Test-Enhanced Learning: Taking Memory Tests Improves Long-Term Retention. Psychological Science, 17(3), 249-255. https://doi.org/10.1111/j.1467-9280.2006.01693.x

JORDAN, K. (2015). Massive open online course completion rates revisited: Assessment, length and attrition. The International Review of Research in Open and Distributed Learning, 16(3). Retrieved from http://www.irrodl.org/index.php/irrodl/article/view/2112

KOLOWICH, S. (2013). The Professors behind the MOOC Hype. Chronicle of Higher Education.

MARGARYAN, A., BIANCO, M., \& LITTLEJOHN, A. (2015). Instructional quality of Massive Open Online Courses (MOOCs). Computers \& Education, 80, 77-83. https://doi.org/10.1016/j.compedu.2014.08.005

TOVEN-LINDSEY, B., RHOADS, R. A., \& LOZANO, J. B. (2015). Virtually unlimited classrooms: Pedagogical practices in massive open online courses. The Internet and Higher Education, 24, 1-12.

https://doi.org/10.1016/j.iheduc.2014.07.001

revue-mediations.teluq.ca | vol. 01, no. 1 